\title{
Efeito da utilização de complemento alimentar em idosos atendidos em um ambulatório na cidade de São Paulo
}

\author{
Effect of use of feeding complement in elderly people attended in an outpatient clinic \\ in São Paulo city, Brazil
}

\section{Resumo}

Objetivo: Verificar o efeito da utilização de complemento alimentar na população idosa atendida pelo setor de nutrição de um ambulatório na cidade de São Paulo, por meio da evolução do peso e do Índice de Massa Corpórea (IMC) desses pacientes. Métodos: A amostra foi composta de idosos com 60 anos ou mais, de ambos os sexos, que fazem uso de complemento alimentar prescrito pelas nutricionistas do setor. As variáveis estudadas foram: gênero, idade, peso, estatura, IMC, patologias mais frequentes, quantidade prescrita do complemento, evolução do peso e do IMC, e intervalo médio entre a primeira consulta e o retorno. A coleta de dados se deu por meio das fichas de atendimento, analisadas no início e após o primeiro retorno de consulta. Foi realizada análise de frequência das variáveis contínuas e categóricas, por meio do teste t-pareado com $p<0,05$. Resultados: Foram analisados os dados de 74 pacientes, sendo 47 mulheres e 27 homens, com idade média de 75,9 \pm 8,3 anos. As patologias mais encontradas foram: hipertensão arterial sistêmica $(55,4 \%)$, dislipidemias $(39,2 \%)$ e diabetes mellitus $(28,4 \%)$. A média de peso dos pacientes foi de 51,4kg na primeira consulta, e de 51,9 kg no retorno $(p=0,025)$. O IMC médio foi de $21,71 \mathrm{~kg} / \mathrm{m}^{2}$ na primeira consulta, e de 21,90 $\mathrm{kg} / \mathrm{m}^{2}$ no retorno $(p=0,040)$. Conclusões: O presente trabalho mostrou que a utilização do complemento alimentar na população de idosos estudada teve impacto positivo, mostrando a importância da intervenção sobre o estado nutricional para a promoção da saúde do indivíduo.

\section{Abstract}

Objective: Verifying the effect of the use of feeding complement on elderly people attended by the nutrition sector of an outpatient clinic in Sao Paulo city, through weight evolution and Body Mass Index (BMI) of these patients. Methods: The sample comprised elderly people aged 60 years or more, from both sexes, who take feeding complement prescribed by the sector nutritionist. The variables studied were genre,

\footnotetext{
Programa de Aprimoramento em Gerontologia Multiprofissional. Instituto Paulista de Geriatria e Gerontologia “José Ermírio de Moraes”. São Paulo, SP, Brasil.

2 Instituto Paulista de Geriatria e Gerontologia “José Ermírio de Moraes”. São Paulo, SP, Brasil.
}

Palavras-chave: Saúde do Idoso. Nutrição do Idoso. Idoso. Suplementação Alimentar. Envelhecimento. 
age, weight, height, BMI, frequent pathologies, amount prescribed by the complement, weight evolution and BMI, certain time between the first appointment and return. Data was collected in attendance records, analyzed in the beginning and after the first return of appointment. Frequency of continuous and categorical variables has been carried out and analyzed through paired-t test with $\mathrm{p}<0.05$. Results: 74 patients records have been analyzed, 47 women and 27 men, with average age of $75.9 \pm 8.3$ years. The most frequent pathologies found were: systemic arterial hypertension, (55.4\%) dislipidemy $(39.2 \%)$ and diabetes mellitus (28.4\%). Weight average of patients has been of $51.4 \mathrm{Kg}$ in the first appointment and $51,9 \mathrm{Kg}$ in the return $(\mathrm{p}=0,025) . \mathrm{BMI}$ average was $21,71 \mathrm{Kg} /$ $\mathrm{m}^{2}$ in the first appointment, and $21,90 \mathrm{Kg} / \mathrm{m}^{2}$ in the return $(\mathrm{p}=0.40)$. Conclusions: This work has shown that the use of feeding complement among elderly people who were studied has succeed, showing the importance of intervention on nutritional situation in order to improve the individual's health.
Key words: Health of the Elderly. Elderly Nutrition. Aged. Supplementary Feeding. Aging.

\section{INTRODUÇÃO}

Apesar de ser um processo natural, o envelhecimento pode submeter o organismo a diversas modificações anatômicas e funcionais, com repercussões nas condições de saúde e nutrição do idoso. ${ }^{1}$ A manutenção da saúde, nos diferentes estágios da vida é influenciada de maneira significativa pela alimentação, que também influenciará a qualidade de vida na senescência. $^{2}$

Os idosos constituem grupo de risco de carência de macro e micronutrientes, pois frequentemente apresentam dificuldades na manutenção adequada da ingestão energética e de nutrientes por meio de uma alimentação balanceada. ${ }^{3}$ Entre as mudanças fisiológicas que interferem no estado nutricional, destacam-se: diminuição do metabolismo basal, redistribuição da massa corporal, alterações no funcionamento digestivo, alterações na percepção sensorial e diminuição da sensibilidade à sede. Com exceção das duas primeiras, todas as outras podem interferir diretamente no consumo alimentar.,

Múltiplos mecanismos levam à ingestão alimentar reduzida no idoso, tais como perda de apetite, redução do paladar e olfato, saúde oral prejudicada, saciedade precoce, além de fatores psicossociais, econômicos e medicamentosos. ${ }^{6}$
Assim como o sobrepeso e a obesidade, o baixo-peso é indicador para diferentes patologias e relaciona-se com importante aumento no risco de morbi-mortalidade. A perda de peso referida pelo paciente deve ser considerada, pois $10 \%$ ou mais de perda de peso, em um período de seis meses, é um provável indicador de problemas de saúde, além de estar associada ao prejuízo no estado nutricional do paciente. Percebe-se que, muitas vezes, a desnutrição é vista tanto pelos profissionais como pelos idosos e seus cuidadores como parte do processo normal de envelhecimento, sendo frequentemente ignorada. ${ }^{8}$

Sousa consideraimportante odesenvolvimento de meios para a recuperação do estado nutricional e o estímulo de uma resposta positiva na saúde dos idosos. ${ }^{9}$ A complementação alimentar é um meio válido e eficaz para recuperar e manter o estado nutricional em idosos. Contribui para adequada vigilância nutricional e manutenção das recomendações diárias para os indivíduos nessa faixa etária. ${ }^{10}$

O presente estudo se propôs a verificar o efeito da utilização de complemento alimentar na população idosa atendida pelo setor de nutrição de um ambulatório na cidade de São Paulo, por meio da evolução do peso e do IMC (Índice de Massa Corpórea) desses pacientes. 


\section{METODOLOGIA}

Trata-se de estudo transversal, realizado no Instituto Paulista de Geriatria e Gerontologia José Ermírio de Moraes (IPGG), um ambulatório de especialidades do governo do Estado de São Paulo, que atende, de forma específica e integral, idosos residentes em regiões periféricas da cidade de São Paulo (São Miguel Paulista, Ermelino Matarazzo, Itaim Paulista, Vila Curuçá), visando promover o envelhecimento saudável. O diferencial do local onde foi feito o estudo é o fornecimento gratuito do complemento alimentar para alguns idosos que são atendidos pelo setor de nutrição. A coleta de dados se deu no período de $1^{\circ}$ a 30 de novembro de 2009.

Informações sobre o estado nutricional do paciente, ou aparente risco nutricional justificado por perda de peso recente, eram utilizadas pelas nutricionistas do setor como critérios para distribuição do complemento, para prescrição da quantidade e tempo de uso do complemento alimentar, pois até o momento da coleta de dados não existia um protocolo estabelecido para definir esses critérios. O complemento utilizado pelo setor tem como definição "produto lácteo, com fonte proteica de alto valor biológico, com prebiótico, enriquecido de vitaminas e sais minerais, em pó" e está acondicionado em embalagem hermeticamente fechada (lata de $400 \mathrm{~g}$ ). Os idosos que recebiam o complemento alimentar eram orientados a utilizá-lo em sua casa, como complemento da sua alimentação, seguindo instruções dadas pela nutricionista que os atendia: diluir duas colheres do produto ( $31,5 \mathrm{~g})$ em 180 $\mathrm{ml}$ de leite de vaca, e tomar de uma a duas vezes ao dia. O complemento alimentar fornecia 230 kcal, quando utilizado uma vez ao dia, e $460 \mathrm{kcal}$, quando utilizado duas vezes ao dia.

A amostra foi composta de idosos com 60 anos ou mais, de ambos os sexos, atendidos pelo setor de nutrição, que fazem uso de complemento alimentar prescrito pelas nutricionistas do setor. Estes idosos foram analisados em conjunto, não havendo separação de grupos conforme a quantidade do suplemento que utilizavam (uma ou duas vezes ao dia). Não houve grupo controle para este estudo. Os idosos foram avaliados com base nos dados fornecidos pelas fichas de atendimento, localizadas no setor de nutrição, que atende atualmente cerca de 960 idosos, porém não todos em uso do complemento alimentar. Os critérios de inclusão foram: idosos que tiveram o início do uso do complemento alimentar no período de janeiro de 2008 a agosto de 2009, além de terem tido acompanhamento regular com o setor de nutrição (retornos a cada 30, 60 ou 90 dias) e possuírem dados de peso e altura para o cálculo do IMC. Os critérios de exclusão foram: idosos que iniciaram o uso do complemento alimentar antes de janeiro de 2008, ou após agosto de 2009; idosos que não tinham dados de peso e altura; idosos que tiveram o retorno com intervalo muito superior quando comparado aos outros pacientes; IMC igual e/ou acima de $28 \mathrm{~kg} / \mathrm{m}^{2}$.

As variáveis estudadas foram: gênero; idade (em anos); peso (em $\mathrm{kg}$ ), aferido por meio de balança, tipo plataforma, da marca Toledo com carga máxima de $150 \mathrm{~kg}$, e intervalos de 50 gramas; estatura (em metros), aferida com antropômetro localizado na mesma balança; IMC (calculado por meio da relação peso em $\mathrm{kg}$ dividido pela altura, em metros, ao quadrado); patologias mais frequentes; quantidade prescrita do complemento; evolução do peso e do IMC; intervalo médio entre a primeira consulta e o retorno. O IMC foi classificado conforme a recomendação da Organização Pan-Americana da Saúde (OPAS), utilizada na pesquisa SABE de 2003, onde: $\mathrm{IMC}<23$ = baixo peso; $23 \leq \mathrm{IMC}$ $<28=$ peso normal; $28 \leq \mathrm{IMC}<30=$ risco de obesidade; IMC $\geq 30=$ obesidade. $^{11}$

O paciente teve seus dados analisados no início do uso do complemento e após o primeiro retorno de consulta, que aconteceu durante o período analisado no estudo. Foi realizada análise de frequência das variáveis contínuas 
e categóricas. Para tanto, foi utilizado o pacote estatístico Statistical Package for the Social Science - SPSS, versão 13.1, comparado ao nível de significância de $p<0,05$. Para a comparação das variáveis entre a avaliação inicial do paciente e a reavaliação, foi feita comparação entre os intervalos de confiança das variáveis analisadas. Analisou-se, por meio do teste $t$-pareado, se houve diferença no peso e IMC no primeiro retorno de consulta após o início do uso do complemento.

O estudo obteve a aprovação do Comitê de Ética em Pesquisa do Instituto de Saúde, sob o parecer $n^{\circ}$ 007/2009. Foi dispensado o Termo de Consentimento Livre e Esclarecido, por se tratar de uma coleta de dados nos prontuários dos pacientes. Para tanto, solicitou-se autorização ao diretor da instituição, com garantia de confidencialidade sobre os usuários.

\section{RESULTADOS}

Foram analisados os dados de 179 pacientes, sendo excluídos 66 que iniciaram o uso do complemento alimentar antes do período estudado, 28 que não tinham dados de peso e altura para o cálculo do IMC, sete que tinham o IMC acima de $28 \mathrm{~kg} / \mathrm{m}^{2}$, e quatro que tiveram o retorno com intervalo muito superior quando comparado aos outros pacientes. Restaram assim, para a comparação das variáveis, 74 pacientes, conforme a tabela 1.

Tabela 1 - Divisão por gênero dos participantes do estudo. São Paulo-SP, 2009.

\begin{tabular}{ccc}
\hline Gênero & $\mathrm{n}$ & $\%$ \\
\hline Masculino & 27 & 36,5 \\
Feminino & 47 & 63,5 \\
Total & 74 & 100 \\
\hline
\end{tabular}

Fonte: IPGG (2009).

A idade média dos idosos foi 75,9 $\pm 8,3$ anos. A média do intervalo entre a primeira consulta e o retorno foi 64,8 dias (cerca de dois meses). A quantidade média prescrita de complemento por dia foi de 1,8 vezes, ou seja, foi prescrito ao paciente fazer uso do complemento alimentar, aproximadamente duas vezes, ou seja, dois copos ao dia.
Em relação ao estado nutricional, no momento da primeira avaliação dos dados, 49 pacientes $(66 \%)$ apresentavam baixo-peso, e 25 (34\%) apresentavam peso adequado, segundo o IMC classificado pela OPAS. ${ }^{11}$ A tabela 2 mostra a evolução do peso dos pacientes após análise dos dados no primeiro retorno dos pacientes após o início do uso do complemento alimentar.

Tabela 2 - Evolução do peso dos participantes do estudo. São Paulo-SP, 2009

\begin{tabular}{ccc}
\hline Evolução do peso $(\mathrm{kg})$ & $\mathrm{n}$ & $\%$ \\
\hline Ganhou & 38 & 51,4 \\
Manteve & 10 & 13,5 \\
Perdeu & 26 & 35,1 \\
Total & 74 & 100 \\
\hline
\end{tabular}

Fonte: IPGG (2009). 
A média de peso dos pacientes foi de $51,4 \mathrm{~kg}$ $\pm 8,3 \mathrm{~kg}$ na primeira consulta, e de $51,9 \mathrm{~kg} \pm 8,7$ $\mathrm{kg}$ no retorno $(p=0,025)$. O IMC médio foi de $21,71 \mathrm{~kg} / \mathrm{m}^{2}$ na primeira consulta, e de $21,90 \mathrm{~kg} /$ $\mathrm{m}^{2}$ no retorno $(p=0,040)$.

As patologias mais encontradas foram: hipertensão arterial sistêmica, em 41 dos pacientes analisados (55,4\%), dislipidemias em 29 pacientes (39,2\%), e diabetes mellitus em 21 pacientes $(28,4 \%)$. Outras patologias foram encontradas, porém em menor proporção, como por exemplo: osteoartrose $(8,1 \%)$, gastrite $(6,7 \%)$, depressão $(6,7 \%)$, hiperuricemia $(5,4 \%)$, e insuficiência coronariana $(4 \%)$.

\section{DISCUSSÃO}

Estudos utilizando o índice de massa corporal (IMC) como uma das formas de avaliar o estado nutricional de idosos têm mostrado preocupantes prevalências de baixo-peso. Em pesquisa realizada com idosos residentes na cidade de São Paulo, verificou-se que 24,1\% deles apresentavam baixo-peso, tendo nos homens maior prevalência $(29,2 \%)$ que nas mulheres $(20,6 \%) .^{12}$

Menezes, avaliando o estado nutricional de idosos residentes em instituições de longa permanência em Fortaleza, observou que 49,8\% dos idosos apresentavam baixo-peso, sendo similar ao presente estudo, que encontrou $66 \%$ dos idosos abaixo do peso adequado. ${ }^{13}$

Estudo da Organização Mundial da Saúde (OMS) realizado em 2005 apontou que homens, a partir dos 65 anos, e mulheres, a partir dos 75 anos, passam a perder peso, devido a alterações fisiológicas como perda de água corporal e redução de peso das vísceras, além da redução de tecido muscular. ${ }^{14}$

A intervenção nutricional em Geriatria deve ser realizada de maneira individualizada, em função do estado nutricional e das múltiplas doenças acometidas, das mudanças biológicas e psicológicas associadas ao envelhecimento, da capacidade funcional e das necessidades nutricionais do indivíduo. Seu objetivo é proporcionar todos os nutrientes necessários em quantidades adequadas, para manter um bom estado nutricional. ${ }^{15}$

Existem diferentes alternativas para melhorar o estado nutricional de pessoas idosas, sendo a maior parte apontada quanto ao consumo alimentar, envolvendo o benefício na quantidade, na qualidade ou a combinação de ambas. A quantidade consumida pode ser influenciada pelas condições de acesso aos alimentos, assistência na alimentação, melhora no ambiente de refeições e sabor das preparações. A qualidade do consumo pode ser aprimorada por meio de orientações nutricionais de uma alimentação balanceada, pelo fornecimento de alimentos variados e nutritivos, e pela verificação da necessidade de complementação nutricional. ${ }^{16}$

Jonhesen e colaboradores, em pesquisa sobre desnutrição em idosos e uso apropriado de complementos alimentares orais, realizada em 2000, referem que o método adequado para minimizar a desnutrição é o uso de alimentos e bebidas pelos pacientes, complementadas com nutrientes específicos, fornecendo melhor quantidade de calorias e maior teor proteico. ${ }^{17}$ É possível que os complementos orais, se bem indicados e adequadamente utilizados, tornem a terapia nutricional altamente especializada, ou no mínimo de mais fácil execução. ${ }^{18}$

O Ministério da Saúde definiu em 1998 que complemento alimentar, ou complemento nutricional, é um produto elaborado com a finalidade de complementar a dieta cotidiana de uma pessoa saudável, que deseja compensar um possível déficit de nutrientes, a fim de alcançar os valores da dose diária recomendada (DDR), não podendo substituir os alimentos, nem se constituir em componente exclusivo da dieta. ${ }^{19}$ É o método mais simples, mais natural e menos invasivo para o aumento na ingestão de nutrientes em todos os pacientes. Deve fornecer quantidades adequadas de todos os nutrientes - proteínas, energia, vitaminas e minerais -, de modo a reforçar as necessidades nutricionais dos pacientes. ${ }^{20}$ 
O presente trabalho mostrou que a utilização do complemento alimentar na população de idosos estudada teve impacto positivo, verificado por meio do ganho de peso e da evolução do IMC. Não foi possível verificar se os idosos que utilizaram o complemento alimentar duas vezes ao dia tiveram ganho de peso maior em comparação aos idosos que utilizaram o complemento apenas uma vez, pois eles não foram analisados separadamente, mas em conjunto.

Resultado semelhante foi obtido por Fernandes em estudo realizado com idosos hospitalizados e desnutridos ou com risco de desnutrição, que comparou os efeitos da complementação alimentar via oral industrializada e lanche equivalente; mostrou que, após a intervenção, os idosos dos dois grupos apresentaram melhora do estado nutricional, de acordo com o método de avaliação utilizado (MAN ${ }^{\circledR}$ - Mini Avaliação Nutricional), favorecendo assim a reabilitação nutricional. ${ }^{21}$

Segundo Volkert, em estudo realizado em 1996, idosos com mais de 75 anos, quando recebem uma complementação calórica durante os períodos de internação e durante a evolução ambulatorial, independentemente da dieta usual, apresentaram nítida melhora das condições gerais, em comparação ao controle, que recebeu somente a dieta usual. ${ }^{22}$

No estudo de Norregaard et al., aplicado em pacientes com doença pulmonar obstrutiva (DPOC) recebendo complementação oral por cinco semanas, foram verificadas melhoras no valor calórico e proteico consumido e na massa corporal, principalmente magra, assim como no funcionamento pulmonar, conforme medido por ventilação voluntária máxima. ${ }^{23}$ No estudo de Potter e colaboradores, que acompanhou 381 idosos recebendo $120 \mathrm{ml}$ de complemento oral hipercalórico e hiperproteico três vezes por dia, verificou-se que esta intervenção proporcionou aumento da ingestão alimentar, aumento de peso corporal, evolução da capacidade funcional e diminuição da taxa de mortalidade dos pacientes investigados. ${ }^{24}$

Arnaud-Battandier et al. mostraram que a utilização de complementação alimentar proporcionou aumento significativo dos nutrientes específicos oferecidos, resultando em ganho de peso corporal e consequentemente melhorando a classificação do estado nutricional avaliado por meio da MAN em idosos desnutridos. ${ }^{25}$ Outro estudo realizado pelo mesmo autor, em 2004, com 378 idosos com idade acima de 70 anos, mostrou que a suplementação oral pode induzir a melhoria do estado nutricional e que o gasto global de recursos com saúde pode ser reduzido. ${ }^{26}$

Ausman \& Russel, em um programa de refeições com base na verificação da utilização de um complemento de polímero líquido durante quatro meses, conseguiram proporcionar aumento de peso na maioria dos pacientes, associado ao aumento da albumina sérica, capacidade total de ligação de ferro, folato, vitamina $\mathrm{C}$ e vitamina $\mathrm{B} 12 .{ }^{27}$

Em estudo de Benamouzign realizado em 2001, a inclusão de alimentos de maior densidade calórica, de alimentos do consumo habitual dos pacientes e ingestão de suplementos proteicos e energéticos, mostrou que esse tipo de suplementação em indivíduos idosos hospitalizados acarreta diminuição do tempo de hospitalização e de complicações, melhorando os parâmetros antropométricos (peso e porcentagem de gordura corpórea). ${ }^{28}$

Gazzotti, em estudo realizado em 2003 com pacientes acima de 75 anos em risco de desnutrição, mostrou que a suplementação nutricional oral promoveu melhoria nos parâmetros antropométricos e biológicos, melhora na pontuação da MAN, juntamente com redução simultânea da morbidade e mortalidade e tempo de permanência hospitalar. ${ }^{29}$

Segundo estudos de Wilson e colaboradores, o consumo de complemento alimentar, quando utilizado no período entre as refeições, no mínimo uma hora antes, não interfere na aceitação das dietas ofertadas e promove a melhora do estado nutricional, considerando o nível de satisfação e saciedade de idosos institucionalizados. ${ }^{30}$

A maioria dos estudos mostra que a utilização de complemento alimentar traz resultados 
positivos para o paciente. A maior parte deles, porém, trata do uso de complementação alimentar feita em âmbito hospitalar, poucos falam do uso do complemento alimentar em idosos em atendimento ambulatorial, o que dificulta comparações.

A complementação alimentar, aliada a um estilo de vida saudável, apresenta um potencial para atenuar as deficiências nutricionais na terceira idade, como também as doenças decorrentes da má nutrição. ${ }^{31}$

No entanto, mesmo diante dos estudos mostrando os benefícios da complementação alimentar em pacientes idosos, é necessário também considerar que, por mais que o valor nutricional dessa complementação seja adequado, ele será de pouca importância se o paciente não aceitá-lo ou se deixar de manter uma ingestão suficiente. ${ }^{32}$ Esta é uma limitação do estudo, pois como o paciente recebe o complemento alimentar para ser utilizado em sua residência, é de fundamental importância

\section{REFERÊNCIAS}

1. Campos MTFS, Monteiro JBR, Ornelas APRC. Fatores que afetam o consumo alimentar e a nutrição do idoso. Rev Nutr 2000;13(3):157-65.

2. Marucci MFN, Alves RP, Gomes MMBC. Nutrição na geriatria. In: Silva SMCS, Mura JDP. Tratado de alimentação, nutrição e dietoterapia. São Paulo: Roca; 2007. p. 391-416.

3. Jensen GL, McGee M, Binkley J. Nutrition in the elderly. Gastroenterol Clin North Am 2001;30(2):313-34.

4. Quintero-Molina R. Nutrición en los ancianos. Geriatrika 1993;9(1):14-8.

5. Nogués R. Factors que afectan la ingesta de nutrientes en el anciano y que condicionan su correcta nutrición. Nutrición Clínica 1995;15(2):39-44.

6. Silva TAA, Junior AF, Pinheiro MM, Szejnfeld VL. Sarcopenia Associado ao Envelhecimento: Aspectos Etiológicos e Opções Terapêuticas. Rev Brás Reumatol 2006;46:391-7.

7. Costa EFA, Monego ET. Avaliação Geriátrica Ampla (AGA). Rev UFG [periódico na internet]. 2003 [acesso [acesso em 4 nov 2009];5(2):[aproximadamente 9 p.]. Disponível em: http://www.proec.ufg.br/ revista_ufg/idoso/aga.html\#IDADE. que ele o faça seguindo corretamente as instruções dadas pela nutricionista que o atende, para que se obtenha o efeito desejado.

\section{CONCLUSÃO}

O presente estudo verificou que a utilização de complemento alimentar na população estudada apresentou resultados positivos no ganho de peso dos idosos e traz a reflexão que investimentos em pesquisas e programas de avaliação e intervenção sobre o estado nutricional podem ser ferramentas importantes na promoção da saúde do indivíduo, visando prevenir ou identificar precocemente deficiências nutricionais e assim tentar minimizar seus efeitos ao longo da vida. No Brasil, entretanto, a literatura disponível até o presente momento, em relação ao uso de complemento alimentar via oral associado à alimentação em idosos, mostrase escassa, justificando assim o desenvolvimento de mais estudos de intervenção alimentar.

8. Jacob Filho W. Envelhecimento e atendimento domiciliário. In: Duarte YAO, Diogo MJD. Atendimento domiciliar: um enfoque gerontológico. São Paulo: Atheneu; 2000. p 19-26.

9. Sousa VMC. Impacto de um produto dietético sobre o estado nutricional em idosos [tese de mestrado] Campinas: Unicamp; 2007.

10. Miguel Mari JA, Pérez del Rio MA. Resultados obtenidos en personas de edad avanzada desnutridas, con la adición a su dieta habitual de un preparado proteico-vitamínico-mineral. Geriatrics 1981;26:50-1.

11. Organización Panamericana de la Salud (OPAS). XXXVI Reunión del Comité Asesor de Investigaciones en Salud. Encuestra Multicéntrica: Salud Beinestar y Envejecimiento (SABE) en América Latina y el Caribe. Informe preliminar. Washington: HPP/OPAS; 2001. [acesso em 4 nov 2009]. Disponível em: http://www.opas.org/program/ sabe.htm. http://www.imsersomayores.csic.es/ documentos/documentos/paho-salud-01.pdf

12. Marucci MFN, Barbosa AR. Estado nutricional e capacidade física. In: Lebrão ML, Duarte YAO, organizadores. SABE - Saúde, Bem-estar e Envelhecimento - O projeto SABE no Município de São Paulo: uma abordagem inicial. Brasília: Organização Pan-Americana da Saúde; 2003. p. 95-117. 
13. Menezes TN. Avaliação antropométrica e do consumo alimentar de idosos residentes em instituições geriátricas da cidade de Fortaleza/Ceará [dissertação de mestrado]. São Paulo: Faculdade de Saúde Pública da Universidade de São Paulo; 2000.

14. Organização Mundial da Saúde. Envelhecimento ativo: uma política de saúde. Brasília: OPAS; 2005.

15. Sousa VMC, Guariento ME. Avaliação do idoso desnutrido. Rev Soc Bras Clin Med 2009;7(1):46-9.

16. Wouters-Wesseling W, Slump E, Kleijer CN, de Groot CPGM, van Staveren WA. Early supplementation after infection in nursing home residents. In: Wouters-Wesseling W. Impact of nutritional supplements on health indices in elderly people. Netherlands: Wageningen University; 2002. p. 27-30.

17. Johnsen C, East JM, Glassman P. Management of malnutrition in the elderly and the appropriate use of commercially manufactured oral nutritional supplements. J Nutr Health Aging 2000;4(1):42-6.

18. Baxter YC, Waitzberg DL. Indicações e uso de suplementos nutricionais orais. In: Waitzberg DL, editor. Nutrição oral, enteral e parenteral na prática clínica. 3. ed. São Paulo: Atheneu; 2001. p. 543-58.

19. Secretaria de Vigilância Sanitária (Brasil). Portaria no 32, de 13 de janeiro de 1998. Institui a necessidade de normatizar o uso de suplementos vitamínicos e ou minerais no país. [acesso em [acesso em 4 nov 2009]. Disponível em: http://www.sfdk.com.br/imagens/ lei/MS\%20-\%20Portaria \%2032.htm.

20. Oliveira T. A importância do acompanhamento nutricional para pacientes com câncer. Prática Hospitalar 2007;9(51):150-4.

21. Fernandes EA. Estudo comparativo dos efeitos de complementação alimentar industrializada e lanche equivalente no estado nutricional de idosos hospitalizados, desnutridos ou em risco de desnutrição [dissertação de mestrado]. São Paulo: Faculdade de Saúde Pública da Universidade de São Paulo; 2008.

22. Volkert D, Hübsch S, Oster P, Schlierf G. Nutritional support and functional status in undernourished geriatric patients during hospitalization and 6-month folow-up. Aging (Milano) 1996;8(6):386-95.
23. Norregaard O, Tottrup A, Saaek. Effects of oral nutritional supplements on adults with chronic obstructive pulmonary disease. Clin Resp Physiol 1987;23(12):338.

24. Potter JF, Schaffer DF, Bohi RL. In-hospital mortality as a function of body mass index: an agedependent variable. J Gerontol 1988;43(3):M59-63.

25. Arnaud-Battandier F Lauque S, Paintin M, Mansourian R, Vellas B, Guigoz Y. MNA and nutrition intervention. In: Mini Nutritional Assesment (MNA): research and pratice in the elderly. Basel: Karger; 1999. p. 131-40.

26. Arnaud-Battandier F, Malvy D, Jeandel C, Schmitt C, Aussage P, Beaufrère B, Cynober L. Use of oral supplements in malnourished elderly patients living in the community: a pharmaco-economic study. Clin Nutr 2004;23(5):1096-103.

27. Ausman LM, Russel RM. Nutrição do idoso. In: Shils ME, Olson JA, Shike M, Ross AC, editores. Tratado de nutrição moderna na saúde e na doença. 9 ed. São Paulo: Manole; 2003. p. 931-43.

28. Bos C, Benamouzig R, Bruhat A, Roux C, Valensi $\mathrm{P}$, Ferrière F, Tomé D. Nutritional status after short-term dietary supplementation in hospitalized malnourished geriatric patients. Clin Nutr 200;20(3):225-33.

29. Gazzotti C, Arnaud-Battandier F, Parello M, Farine S, Seidel L, Albert A, Petermans J. Prevention of malnutrition in older people during and after hospitalization: results from a randomised controlled clinical trial. Age Ageing 2003;32(3):321-5.

30. Wilson MM, Purushothaman R, Morley JE. Effect of liquid dietary supplements on energy intake in the elderly. Am J Clin Nutr 2002;75(5):944-7.

31. Novaes MRCG, Ito MK, Arruda SF, Rodrigues P, Lisboa AQ. Suplementação de micronutrientes na senescência: implicações nos mecanismos imunológicos. Rev Nutr 2005;18(3):367-76.

32. Schiffman SS, Wedral E. Contribution of taste and smell losses to the wasting syndrome. Age Nutr 1996;7:106-20. 